\title{
Vitamin D Supplementation - Still in its Infancy
}

\author{
Tim CheethaM \\ Paediatric Endocrinology, Institute of Human Genetics, Newcastle University; and Department of Paediatrics, Royal Victoria \\ Infirmary Newcastle upon Tyne, NE1 4LP, UK.T.D.Cheetham@newcastle.ac.uk
}

$\mathrm{M}$ any healthcare professionals have vitamin $\mathrm{D}$ on their minds at the moment and those that aren't thinking about it may need to in the near future. The fact that many people are vitamin D insufficient has been highlighted by studies in populations from Western Europe but also areas perceived to have sunnier climates such as North America, Africa and Australia. Dark skin generates a fraction of the amount of vitamin D generated by fair skin when exposed to sunlight and is undoubtedly a risk factor for a low vitamin D status. Traditional dress, time spent indoors rather than outdoors and a low dietary intake of foods containing vitamin $\mathrm{D}$ has also been implicated. It is therefore important to emphasize that people from broad sections of society may be at risk, irrespective of skin color(1). With this background it is surprising that the supplementation of at risk groups such as pregnant women and children with vitamin $\mathrm{D}$ has not been given greater priority in many countries. Obstetricians, midwifes, neonatologists and pediatricians have, it can be argued, been a little slow to address the suboptimal vitamin D status of many of our patients. The potential link between a low vitamin D status and cardiovascular disease, diabetes and cancer means that other health professionals may have to take note now as well(2-4).

The well-designed article by Mehrotra and colleagues from Delhi(5) is yet another reminder of how important vitamin D status is in determining the well-being of pregnant women and their offspring. The fact that so many infants with a suboptimal vitamin D status develop hypocalcemic seizures is quite alarming. Many health professionals know about vitamin D insufficiency and skeletal deformity but seizures in the first weeks of life must clearly result in an assessment of calcium concentrations straight away. Seizures as a presentation of vitamin $\mathrm{D}$ deficiency seems to be much more common than people previously thought. Other notable aspects of Mehrotra and colleagues' study include the close relationship between vitamin $\mathrm{D}$ status in mother and child. Furthermore, the difference in calcium intake between patients and controls suggests that a low calcium intake might also contribute to risk of seizures(6). A potential 'catch' for doctors investigating babies with vitamin D deficiency is the fact that parathormone (PTH) levels are frequently not elevated in contrast to older children.

What should we do? Well, the first issue is to target high risk groups such as pregnant women and the rapidly growing child. Vitamin D supplementation can be given relatively easily in the form of a 'children's multi-vitamin' supplement. Vitamin D2 (ergocalciferol) or D3 (choleclalciferol) should be provided to pregnant women. It is important to highlight the fact that 1-alphacalcidol is not an effective therapy for vitamin D deficiency and hence should not be used in this context. We should all be made aware of the need for calcium containing foods and the beneficial role of sunlight. Too much sun and associated skin erythema is not good for health but some sun exposure is an effective way of enhancing vitamin D status. Hence sunlight is like so many things in life - too much may not be good for you but too little may not be good for you either. For people who cannot easily increase sunlight exposure 
or increase their oral intake of vitamin D with foods such as oily fish, long term vitamin D2 or D3 supplementation will be appropriate.

Funding: None.

Competing interests: None stated.

\section{REFERENCES}

1. Beck-Nielsen SS, Jensen TK, Gram J, Brixen K, Brock-Jacobsen B. Nutritional rickets in Denmark: A retrospective review of children's medical records from 1985 to 2005. Eur J Pediatr 2009; 168: 941-949.

2. Dobnig H, Pilz S, Scharnagl H, Renner W, Seelhorst U, Wellnitz B, et al. Independent association of low serum 25-hydroxyvitamin $\mathrm{D}$ and 1,25-dihydroxyvitamin D levels with all-cause and cardiovascular mortality. Arch Intern Med 2008; 168: 1340-1349.
3. Zipitis CS, Akobeng AK. Vitamin D supplementation in early childhood and risk of type 1 diabetes: A systematic review and meta-analysis. Arch Dis Child 2008; 93: 512-517.

4. Lappe JM, Travers-Gustafson D, Davies KM, Recker RR, Heaney RP. Vitamin D and calcium supplementation reduces cancer risk: Results of a randomized trial. Am J Clin Nutr 2007; 85: 15861591.

5. Mehrotra P, Marwaha RK, Aneja S, Seth A, Singla BM, Ashraf G, et al. Hypovitaminosis D and hypocalcemic seizures in infancy. Indian Pediatr 2010; 47: 581-586.

6. DeLucia MC, Mitnick ME, Carpenter TO. Nutritional rickets with normal circulating 25hydroxyvitamin D: A call for re-examining the role of dietary calcium intake in North American infants. J Clin Endocrinol Metab 2003; 88: 35393545 . 